\title{
General Quantum Theory No Axiom Presumption: I \\ ----Quantum Mechanics and Solutions to Crisises of Origins of Both Wave-Particle Duality and the First Quantization
}

\author{
C. Huang ${ }^{1,2, *}$, Yong-Chang Huang ${ }^{3,4,+}$ and Jia-Min Song ${ }^{3,++}$ \\ 1. Lawrence Berkeley National Laboratory, 1 Cyclotron Road, Berkeley CA 94720, USA \\ 2. Department of Physics and Astronomy, Purdue University, 525 Northwestern Avenue, W. \\ Lafayette, IN 47907-2036, USA \\ 3. Institute of Theoretical Physics, Beijing University of Technology, Beijing, 100124, China \\ 4. Institute of Theoretical Physics, Jiangxi Normal University, Nanchang, 330022, China
}

\begin{abstract}
Density distribution function of classical statistical mechanics is generally generalized as a product of a general complex function and its complex Hermitian conjugate function, and the average of classical statistical mechanics is generalized as the average of the quantum mechanics. Furthermore, this paper derives three ones of the five axiom presumptions of quantum mechanics, e.g., deduces Schrödinger equation by two general ways, makes the three axiom presumptions into three theorems of quantum mechanics, not only solves the crisis to hard understand, but also gets new theories and new discoveries, e.g., this paper solves the crisis of the origin of the wave-particle duality (i.e., complementary principle), derives operators, eigenvalues and eigenstates, deduces commutation relations for coordinate and momentum as well as the time and energy, and discovers quantum mechanics is just a generalization ( mechanics ) theory of the complex square root of ( real density function of ) classical statistical mechanics, which will make people renew thinking modern physics development. In addition, this paper discovers the reason that Schrödinger equation doesn't takes the time derivative of space coordinates. Therefore, this paper gives solution to the Crisis of the first quantization origin.

Key words: Quantum mechanics, operators, basic presumptions, wave-particle duality, the first quantization, commutation relation, quantum communication, classical statistical mechanics, classical mechanics
\end{abstract}

\section{I.Introduction}

As far as quantum mechanics is concerned, there are still many problems that are very difficult to understand in terms of quantum mechanics. But classical mechanics is very well understood, and there is a very close correspondence between classical mechanics and quantum mechanics. Therefore, the studies on the correspondence between classical mechanics and quantum mechanics enable people to have a deeper understanding of quantum mechanics. Since quantum mechanics is a statistical theory [1,2], thus we naturally consider the correspondence relations between classical statistical mechanics and quantum mechanics.

In fact, in the history of physics, people first studied classical mechanics [3], and then further researched on classical statistical mechanics with uncertainty [4], and finally have developmentally investigated to quantum mechanics [5,6], which is the inevitable result of steady development step by step. So, when we think about quantum mechanics, we need to think about the classical limit of quantum mechanics, which is to go first to classical statistical mechanics, and

*Email: c.huang0@ hotmail.com; +email: ychuang@bjut.edu.cn; ++email: jiaminsong@outlook.com 
then to classical mechanics, not to go straight to classical mechanics. Otherwise, it will lead to that there are many problems that are hard to understand, which will lead to some crisises in the interpretations of the bases of current quantum mechanics [7,8]. And a lot of famous pioneers have done a lot of exploration works, but still cannot solve the crisises, for example, the argument between Einstein and Bohr about the interpretation of quantum mechanics [9-12].

In fact, in both classical statistical mechanics and quantum mechanics, there are not only statistical average relations, but also uncertain relations etc. [13]. Therefore, it is necessary to study how the classical statistical mechanics can be directly extended to the situation of quantum mechanics, this paper intends to do just so.

It is now known that the uncertainty relations in both classical statistical mechanics and quantum mechanics are the important relations $[13,14]$. In quantum mechanics, the uncertainty relation is derived from the wave-particle duality of microscopic particles. It is of great practical significance to clarify the correspondence between classical statistical mechanics and quantum mechanics for us to truly understand the more general physical essence of quantum mechanics, especially for the more general connection via quantum mechanics between the stability and uncertainty of the world $[13,15]$.

In general, there is a correspondence between the general statistical sample space of classical statistical mechanics and the Hilbert space of quantum mechanics. Quantum mechanics is a general theory, and classical mechanics can be regarded as the limit of quantum mechanics when quantum effects are ignored. Therefore, there is a correspondence between quantum mechanics and classical mechanics. In classical mechanics, the motion of a particle is determined by the solution of Newton's equations and initial and boundary conditions. If the initial and boundary conditions are completely given, we get a completely deterministic description. If the initial and boundary conditions are given in the form of probability, the solution must also appear in the form of probability, so the form of motion obtained is also in the form of probability. Therefore, the description of classical statistical mechanics can be obtained [16].

The objects described by classical statistical mechanics can be an ensemble or some tiny particles [17]. For a system in classical statistical mechanics, a corresponding relationship with quantum mechanics can be established.

As we all know, quantum mechanics is based on the five basic axiom presumptions: wave function axiom presumption, operator axiom presumption, measurement axiom presumption, evolution axiom presumption and identical axiom presumption, using the five axiom presumptions, one can deduce quantum mechanics $[15,18]$.

We now process from classical statistical mechanics without the basic presumptions to naturally deduce the present quantum mechanics, give its solutions to the two crisises of both wave-particle duality origin and the first quantization interpretations. In addition, new important discoveries were made, and all the achieved conclusions satisfy that the classical limit of quantum mechanics is classical statistical mechanics, and the macroscopic limit of classical statistical mechanics is classical mechanics, which are exactly required by the self-consistency of physics, and are also a flashback of the related cognitive development processes in physics.

After having tried to make a lot of efforts and experienced various explorations following the pioneers' steps [19-22] and up to now various efforts of almost all quantum theorists in quantum theory [23-31], we finally overcome these large difficulties.

Especially, development of quantum Physics was reviewed [32], probability, information and 
statistical physics were discussed [33], furthermore, fundamental principles of theoretical physics, concepts of quasiaverages, quantum protectorate and emergence were systematically studied [34].

The arrangement of this paper is as follows: section 2 studies the general average representation and the related discussions of any observable physical quantity; section 3 shows the general eigen-equations and new general Hellmann-Feynman theorem as well as the related discussions; section 4 deduces the general Schrödinger equation in quantum mechanics and shows the related discussions; section 5 gives applications to different commutation relations and the first quantization; Section 6 is the summary and conclusions.

\section{The general average representation and the related discussions of any observable physical quantity}

In current quantum mechanics, the first basic axiom presumption is the wave function axiom presumption: the motion state of microscopic particles is described by the wave function (material wave), and the modulus square of the wave function is the probability density function of particles. Now we don't need the wave function axiom presumption and are able to derive both wave function axiom presumption content and new more physical contents.

First, considering a general classical statistical physical system, in which a particle has a classical distribution density function $\rho=\rho(\vec{r}, t)$ at any time $\mathrm{t}$, according to the positive definition of classical distribution density function, there is $0 \leq \rho(\vec{r}, t) \leq 1$, and since it must be in the space $\mathrm{V}$ at any time, thus we have $\int_{V} \rho(\vec{r}, t) d \vec{r}=1$, thus the average of any observable physical quantity satisfying the statistical classical mechanics of the particle is

$$
\bar{F}(t)=\int_{V} F(\vec{r}, t) \rho(\vec{r}, t) d \vec{r} .
$$

So, we can give a description of the statistical property of a particle and in order to generalize to the general quantum theory from the classical statistical mechanics that we have, we can consider the density function of any positive definite real number $\rho=\rho(\vec{r}, t)$, mathematically the density function can always be represented as the product of a complex number and its complex Hermitian conjugate

$$
\rho(\vec{r}, t)=\psi *(\vec{r}, t) \psi(\vec{r}, t) .
$$

In order to do general research, we can generally consider when the density function takes discrete values $\rho(\vec{r}, t)=\sum_{i=1}^{n} \rho_{i}(\vec{r}, t)$, then we have $\rho(\vec{r}, t)=\sum_{i=1}^{n} \psi_{i} *(\vec{r}, t) \psi_{i}(\vec{r}, t)$, this expression can be expressed as a matrix product $\rho(\vec{r}, t)=\psi^{+}(\vec{r}, t) \psi(\vec{r}, t)$, where $\psi^{+}(\vec{r}, t)=\left(\psi_{1}^{*}(\vec{r}, t) \psi_{2} *(\vec{r}, t) \ldots \psi_{n}^{*}(\vec{r}, t)\right)$ is the row matrix, which is the Hermitian conjugate of the column matrix $\psi(\vec{r}, t)$. The general average value (2.1) of discrete values of 
observable physical quantities can be expressed again as

$$
\begin{aligned}
& \bar{F}(t)=\int_{V} \sum_{i=1}^{n} F_{i}(\vec{r}, t) \psi_{i}^{*}(\vec{r}, t) \psi_{i}(\vec{r}, t) d \vec{r} \\
&=\int_{V} \psi^{\dagger}(\vec{r}, t) \hat{F}(\vec{r}, t) \psi(\vec{r}, t) d \vec{r} \\
&=\int_{V} \psi^{\prime \dagger}(\vec{r}, t) \hat{F}^{\prime}(\vec{r}, t) \psi^{\prime}(\vec{r}, t) d \vec{r} \\
&=\sum_{m, n=1}^{k} \int_{V} \psi_{m} *(\vec{r}, t) \hat{F}_{m n}^{\prime}(\vec{r}, t) \psi_{n}^{\prime}(\vec{r}, t) d \vec{r}
\end{aligned}
$$

where $\hat{F}(\vec{r}, t)=\operatorname{diag}\left(F_{1}(\vec{r}, t), F_{2}(\vec{r}, t), \ldots, F_{n}(\vec{r}, t)\right)$ is a nxn diagonal square matrix ( without differentiation ) operator of classical general functions; for the third equality, according to linear algebra theory, a general unitary matrix $U(\vec{r}, t)$ satisfies the properties $U^{+}(\vec{r}, t) U(\vec{r}, t)=1 \quad$ ( there is $U^{+}(\vec{r}, t)=U^{-1}(\vec{r}, t)$ ), and we have defined $\psi^{\prime}(\vec{r}, t)=U(\vec{r}, t) \psi(\vec{r}, t)$, and then we have $\psi^{\prime+}(\vec{r}, t)=\psi^{+}(\vec{r}, t) U^{+}(\vec{r}, t)$ and

$$
\hat{F}^{\prime}(\vec{r}, t)=U(\vec{r}, t) \hat{F}(\vec{r}, t) U^{+}(\vec{r}, t),
$$

that is, the off-diagonal square matrix operator can be obtained by a general diagonal square matrix operator $\hat{F}(\vec{r}, t)$ through the similarity transformation of a general unitary operator, in other words, $\hat{F}^{\prime}(\vec{r}, t)$ is a nxn general off-diagonal square matrix operator. In fact, formula (2.4) is an inverse operation on a general diagonalization of a matrix, and it is easy to prove that the general operator (2.4) must be Hermitian, that is, we quite naturally prove that the classical observable operator must be Hermitian.

Therefore, we not only realize from the classical average representation to the quantum mechanics average representation of the operator that is between the probability function and its complex conjugate function, but also naturally find the average representation of different operators can be different in a similarity transformation, but its average value is invariant. And it has been proved in linear algebra that the Hermitian matrix is diagonalizable, and this diagonalizability is the necessary and sufficient condition for the Hermitian matrix to have eigenvalues on its eigenvectors [15].

Therefore, the above research is consistent with the known quantum mechanics theory, which is an important reason why we can generalize from classical statistical mechanics to quantum mechanics. It can be seen, from above discussions, that the three expressions in eq.(2.3) are equivalent invariant expressions, and we show that the density is invariant under this transformation

$$
\rho^{\prime}(\vec{r}, t)=\psi^{\prime+}(\vec{r}, t) \psi^{\prime}(\vec{r}, t)=\psi^{+}(\vec{r}, t) U^{+}(\vec{r}, t) U(\vec{r}, t) \psi(\vec{r}, t)=\rho(\vec{r}, t)
$$

Therefore, we have obtained the general expression (2.3) of the average value of discrete observable values of any physical quantity in classical statistical mechanics, which is the same as 
that of quantum mechanics. These are just the requirements of physics consistence. For any $m$ and n, when there are $\hat{F}_{m n}^{\prime}(\vec{r}, t)=1$, the last equation of eq.(2.3) can be written in normalized form

$$
\bar{F}(t)=\sum_{m, n=1}^{k} \int_{V} \psi_{m}{ }^{\prime+}(\vec{r}, t) \psi^{\prime}{ }_{n}(\vec{r}, t) d \vec{r}=\sum_{m, n=1}^{k} \int_{V} \rho^{\prime}{ }_{m n}(\vec{r}, t) d \vec{r}=1,
$$

where we have used $\psi^{\prime}(\vec{r}, t)=U(\vec{r}, t) \psi(\vec{r}, t)$.

On the other hand, if we take the Hermitian conjugate of equation (2.3), we have

$$
\bar{F}^{+}(t)=\int_{V} \psi^{\prime^{+}}(\vec{r}, t) \hat{F}^{\prime+}(\vec{r}, t) \psi^{\prime}(\vec{r}, t) d \vec{r}
$$

Every physical quantity measured is a real value, thus we have $\bar{F}(t)=\bar{F}^{+}(t)$, using eq.(2.3)

and (2.7), one has $\hat{F}^{\prime}(\vec{r}, t)=\hat{F}^{++}(\vec{r}, t)$, i.e., from an experimental observation point of view, all operators of observable quantities are Hermitian operators, and their eigenvalues are real.

In current quantum mechanics, the second basic axiom presumption is the axiom presumption of operators [15,18]: Classical mechanical quantities correspond to operators of quantum mechanics, the eigenvalue of operators is the measured value of their mechanical quantities. Quantization is the operatorization of mechanical quantities, all the eigenfunctions of quantum mechanics operators are complete.

In addition, we have proved the operators of quantum mechanics correspond to the observable physical quantities in classical statistical mechanics by the generalized expression of operator mean value (2.3), and the eigenvalues of the operators are the measured values of its mechanical quantities. Quantization is the operatorization of mechanical quantities, because eq.(2.3) is the generalized expression from the mean value of observable physical quantities in classical statistical mechanics to the mean value of operators, and the eigenfunctions of all operators of mechanical quantities in quantum mechanics are complete (Since the sample space of classical statistics is not only complete but also corresponding to the eigenfunctions of all quantum mechanical operators). So, we naturally derive the second axiom presumption that is the axiom presumption of operators.

Mathematically we have implemented the sum of observable values from the classical statistical mean value sum to the mean value sum of the quantum mechanics' matrix operators in the middle of a state vector and its complex conjugate. In fact, when we take the state of the microscopic particle system described by the general complex function of square root of the density function, we begin to enter a more general description system that is different from the classical statistical mechanics system, namely the quantum mechanics system.

Therefore, we not only obtain the statistical description of microscopic particles, but also obtain the state vector description of microscopic particles, namely the probability vector description, and obtain the coherence (2.6) of different components of the state vector. Later, we can more specifically prove that this description system is exactly the system of quantum mechanics.

\section{Derivations of general eigen-equations and general Hellmann-Feynman}




\section{theorems}

Following the studies in literature $[15,18]$ and using the generally deduced expression (2.3), we derive the general eigen-equation and general Hellmann-Feynman theorem.

In order to make general studies without losing generality, in eq.(2.3), respectively denoting $\psi^{\prime}$ as $\phi$, then $\psi^{\prime+}=\phi^{+}$, and taking the operator $\hat{F}^{\prime}$ as $\hat{A}$, then eq.(2.3) can be written as

$$
\overline{\hat{A}}=\int \phi^{+} \hat{A} \phi d \tau
$$

Obviously, eq.(3.1) is a direct extension of eq.(2.3), $d \tau$ can be the measure of general representation, that is, $d \tau$ may be taken as the measures of different representations, such as the measures of coordinate, momentum and energy representations etc., and $\int \phi^{+} \phi d \tau=1$ can be obtained under the corresponding measures.

Because according to the probability theory, any particle must have the value under the corresponding measure, so it has its normalization. On the other hand, for the general function $\lambda_{i}(i=1,2, \ldots, n)$, we generally have

$$
\sum_{i=1}^{n} \lambda_{i} \int \phi_{i}^{+} \phi_{i} d \tau=\int \phi^{+} \lambda \phi d \tau
$$

where $\lambda$ is a diagonal matrix. According to the variational theory, $\lambda_{i}(i=1,2, \ldots, n)$ can be taken as the Lagrange multipliers, so the following function can be constructed

$$
\overline{\hat{A}^{\prime}}=\int \phi^{+} \hat{A} \phi d \tau \pm \sum_{i=1}^{n} \lambda_{i} \int \phi_{i}^{+} \phi_{i} d \tau .
$$

Using the variational theory, we have

$$
\begin{aligned}
\delta \hat{A}^{\prime} & =\delta\left(\int \phi^{+} \hat{A} \phi d \tau \pm \sum_{i=1}^{n} \lambda_{i} \int \phi_{i}^{+} \phi_{i} d \tau\right)=0 \\
& =\int \delta \phi^{+}(\hat{A} \pm \lambda) \phi d \tau+\int[(\hat{A} \pm \lambda) \phi]^{+} \delta \phi d \tau+\int \phi^{+}(\delta \hat{A} \pm \delta \lambda) \phi d \tau
\end{aligned}
$$

According to eq.(3.4) and using the independences of complex functions $\delta \phi^{+}$and $\delta \phi$, we have

$$
\begin{gathered}
(\hat{A} \pm \lambda) \phi=0, \text { i. e., } \hat{A} \phi=\mp \lambda \phi, \\
{[(\hat{A} \pm \lambda) \phi]^{+}=0, \text { i. e., }(\hat{A} \pm \lambda) \phi=0}
\end{gathered}
$$

It follows from eq.(3.6) that $\hat{A} \phi=\mp \lambda \phi$, when the positive signs of eqs.(3.5) and (3.6) are taken, the eigenvalue equation in quantum mechanics can be obtained, and it is also necessary to satisfy the condition that the first order linear eq.(3.5) has a non-trivial solution 


$$
|\hat{A}-\lambda|=0 \quad, \quad \lambda=\operatorname{diag}\left(\lambda_{1}, \lambda_{2}, \ldots, \lambda_{n}\right)
$$

Eq.(3.7) is the condition that any matrix in linear algebra can be diagonalized, or the condition that any matrix in linear algebra can be diagonalized is that the matrix has $\mathrm{n}$ linearly independent eigenvectors.

They also indicate that the eigenvalues of all operators are the values corresponding to the extreme values of the variational (3.4). From the last term in eq.(3.4), we have

$$
\int \phi^{+}(\delta \hat{A} \pm \delta \lambda) \phi d \tau=0, \text { i. e. , } \int \phi^{+} \delta \hat{A} \phi d \tau=\mp \int \phi^{+} \delta \lambda \phi d \tau
$$

Using $\delta \lambda=\frac{\delta \lambda}{\delta a_{i}} \delta a_{i}, \delta \hat{A}=\frac{\delta \hat{A}}{\delta a_{i}} \delta a_{i}$ and when $\delta a_{i}$ are linearly independent parameters, eq.(3.8) becomes

$$
\int \phi^{+} \frac{\delta \hat{A}}{\delta a_{i}} \phi d \tau=\int \phi^{+} \frac{\delta \lambda}{\delta a_{i}} \phi d \tau,
$$

where, the positive sign has been taken in eq.(3.8), that is, only the negative sign is taken in eq.(3.3). Therefore, we get the general Hellmann-Feynman theorem (3.9). When the diagonal elements of the matrix are all the same, eq.(3.9) is simplified to the usual Hellmann-Feynman theorem

$$
\frac{\delta \lambda}{\delta a_{i}}=\frac{\int \phi^{+} \frac{\delta \hat{A}}{\delta a_{i}} \phi d \tau}{\int \phi^{+} \phi d \tau}=\left\langle\frac{\delta \hat{A}}{\delta a_{i}}\right\rangle
$$

Eq.(3.9) is the general Hellmann-Feynman theorem that we derived. This relation implies that ordinary Hermitian operators satisfy this important relation as well. In particular, the Hellmann-Feynman theorem is established under the condition that not only the eigenvalues of all operators are the values corresponding to the extreme values of their variational system, but also the values of the rest of the system are the results of taking the extreme values for the variational system.

As a result, we find that not only all the eigenvalues of the operators are the values corresponding to their variational system's taking extreme value, and also find Hellmann-Feynman theorem is established on condition that not only all the eigenvalues of the operators are the values corresponding to extremum value of their variational system, but also the rests of this system are also the result of taking extreme values for the variational system. That is, the whole variational system takes the extreme value among all possible values, i.e., the particle system will choose the optimal way to express the physical properties of the system. More detailed studies are shown in the next section.

\section{Derivation of general Schrödinger equation in quantum mechanics}

Using the general eigen-equation (3.5) derived from us, taking $\hat{A}$ and $\lambda$ as the classical 
Hamiltonian operator $\hat{H}$ and the energy eigenvalue $\mathrm{E}$ ( there is no differential operator but can include the matrix ), respectively, then the equation (3.5) can be specifically written as the corresponding eigen-equation

$$
\hat{\mathrm{H}}(T, V) \phi(\vec{p}, E)=\hat{\mathrm{H}}(\vec{p}, \vec{r}) \phi(\vec{p}, E)=E \phi(\vec{p}, E) .
$$

Namely, in $\mathrm{E}=\int \phi(\vec{p}, E)^{\dagger} \hat{\mathrm{H}}(\vec{p}, \vec{r}) \phi \mathrm{q} .(3.3), d \tau$ can be taken as the measure of momentum representation, i.e., $\overline{\hat{\mathrm{H}}}^{\prime}(T, V)=\int \phi(\vec{p}, E)^{\dagger} \hat{\mathrm{H}}(T, V) \phi(\vec{p}, E) d \vec{p} \quad-\int \phi(\vec{p}, E)^{\dagger} E \phi(\vec{p}, E) d \vec{p}$ $=\int \phi(\vec{p}, E)^{\dagger} \hat{\mathrm{H}}(\vec{p}, \vec{r}) \phi(\vec{p}, E) d \vec{p}-\int \phi(\vec{p}, E)^{+} E \phi(\vec{p}, E) d \vec{p}$. The 4-dimensional momentum representation wave function $\phi(\vec{p}, E)$ just reflects the system's global property (having nothing to do with spacetime coordinates) that is just particle property for the single particle. Using $e^{-i t E / \alpha} /(2 \pi \alpha)^{1 / 2}$ times eq.(4.1) and making the integral from minus infinity to positive infinity, namely, we make Fourier integral transformation of plane wave relevant to energy, we get

$$
\begin{aligned}
& \frac{1}{(2 \pi \alpha)^{1 / 2}} \int_{-\infty}^{\infty} \hat{\mathrm{H}}(\vec{p}, \vec{r}) \phi(\vec{p}, E) e^{-i t E / \alpha} d E=\frac{1}{(2 \pi \alpha)^{1 / 2}} \int_{-\infty}^{\infty} E \phi(\vec{p}, E) e^{-i t E / \alpha} d E \\
& =i \alpha \frac{\partial}{\partial t} \varphi(\vec{p}, t)=\hat{\mathrm{H}}(\vec{p}, \vec{r}) \varphi(\vec{p}, t)
\end{aligned}
$$

where $\alpha$ is a constant making their dimensions $[t E] /[\alpha]=1$, and according to general mathematical principle, we have defined

$$
\varphi(\vec{p}, t)=\frac{1}{(2 \pi \alpha)^{1 / 2}} \int_{-\infty}^{\infty} \phi(\vec{p}, E) e^{-i t E / \alpha} d E
$$

eq.(4.3) is Fourier transformation of plane wave relevant to energy,

Eq.(4.2)'s second line is still the incomplete Schrödinger equation for the classical Hamiltonian $\hat{\mathrm{H}}(\vec{p}, \vec{r})$ and the wave function $\varphi(\vec{p}, t)$ of the momentum representation, Because $\vec{p}$ is still a classical quantity not an operator. And from eq.(4.2), we get $\hat{\mathrm{H}}(\vec{p}, \vec{r})=i \alpha \partial / \partial t$. It can be found from the above study, it is the presence of a weight factor $e^{-i t E / \alpha}$ that enables the classical Hamiltonian to be written in operator form $\hat{\mathrm{H}}(\vec{p}, \vec{r})=i \alpha \partial / \partial t$.

It is well known from the mathematical properties of the Fourier transformation that any continuous physical quantity can be represented by the discrete Fourier basic vector expansion, 
which can be equivalent to the projection of the physical quantity onto the discrete Fourier basic vector, i.e., the physical quantity is thus discretization (i.e., a discrete quantization).

Similarly, any continuous quantity can be represented by a continuous Fourier basic vector expansion. In particular, it should be pointed out that eq.(4.3) uses $e^{-i t E / \alpha} /(2 \pi \alpha)^{1 / 2}$ times $\phi(\vec{p}, E)$ and makes Integral from minus infinity to positive infinity for $\mathrm{E}$, that is, the wave function of the energy representation is deduced at time $t$ via considering the E's contribution to $\phi(\vec{p}, E)$ from minus infinity to positive infinity, which is also the natural property of the Fourier integral transformation for plane wave energy part.

Substituting the classical operator $\hat{\mathrm{H}}(\vec{p}, \vec{r})=T(\vec{p})+V(\vec{r})$ into eq.(4.2), using $e^{i p \cdot r \cdot \beta} /(2 \pi \beta)^{1 / 2}$ to multiply the second row of eq.(4.2), and integrating from minus infinity to positive infinity, then we deduce

$$
\begin{aligned}
& \frac{1}{(2 \pi \beta)^{1 / 2}} \int_{-\infty}^{\infty}[T(\vec{p})+V(\vec{r})] \varphi(\vec{p}, t) e^{i \vec{p} \cdot \vec{r} / \beta} d \vec{p} \\
= & \frac{1}{(2 \pi \beta)^{1 / 2}} \int_{-\infty}^{\infty}[\hat{T}(-i \beta \nabla)+V(\vec{r})] \varphi(\vec{p}, t) e^{i \vec{p} \cdot \vec{r} / \beta} d \vec{p} \\
& =i \alpha \frac{\partial}{\partial t} \psi(\vec{r}, t)=\hat{\mathrm{H}}(\hat{\vec{p}}, \vec{r}) \psi(\vec{r}, t)
\end{aligned}
$$

where we have already used that the kinetic energy $T(\vec{p})$ can be expanded into a series of $\vec{p}$ (e.g., $\left.T(\vec{p})=\vec{p}^{2} /(2 m)\right), \quad \beta$ is a constant making their dimensionality $[\vec{p} \cdot \vec{r}] /[\beta]=1$, and according to general mathematical rule, we can define

$$
\psi(\vec{r}, t)=\frac{1}{(2 \pi \beta)^{3 / 2}} \int_{-\infty}^{\infty} \varphi(\vec{p}, t) e^{i \vec{p} \cdot \vec{r} / \beta} d \vec{p}
$$

Eq.(4.5) is the Fourier transform of momentum part of plane wave $\varphi(\vec{p}, t), \quad \psi(\vec{r}, t)$ is deduced from the probability density of the classical particle and the projection of the time part state vector $\varphi(\vec{p}, t)$ of the base vector of the plane wave to the space part $e^{i \vec{p} \cdot \vec{r} / \beta}$ of the base vector of the plane wave and by integration for momentum $\vec{p}$, this makes it not only have the characteristics of the probability density state vector of the classical particle but also have the characteristics of the plane wave, which makes $\psi(\vec{r}, t)$ have the characteristics of the wave-particle duality state vector at the same time.

The Hamiltonian becomes an operator with differentiation form $\hat{\mathrm{H}}(\hat{\vec{p}}, \vec{r})=\hat{T}(\hat{\vec{p}})+V(\vec{r})$ 
$(\hat{\vec{p}}=-i \beta \nabla)$, it can be found from the above study, it is the presence of a weight factor $e^{i \vec{p} \cdot \vec{r} / \beta}$ that enables momentum $\vec{p}$ to be written in operator form $\hat{\vec{p}}=-i \beta \nabla$. By using eq.(4.4) in a practical example or in comparison with the existing Schrödinger equation, we get $\alpha=\beta=\hbar$, and substituting $\alpha=\beta=\hbar$ into eq.(4.4), we finally get the Schrödinger equation in quantum mechanics

$$
i \hbar \frac{\partial}{\partial t} \psi(\vec{r}, t)=\hat{\mathrm{H}}(\hat{\vec{p}}, \vec{r}) \psi(\vec{r}, t)
$$

Finally, from eq.(4.6), we get that the Hamiltonian operator can be written as $\hat{\mathrm{H}}(\hat{\vec{p}}, \vec{r})=i \hbar \partial / \partial t$.

It can be seen from the above derivation that eq.(4.1) is the eigenvalue equation of the probability of classical particles from classical statistical mechanics, and the state function $\phi(\vec{p}, E)$ is the complex square root of the probability density of classical particles in the complex number field, $\vec{p}, E$ are the physical quantities of classical particles in classical statistical mechanics, and they reflect the properties of particles.

When eq.(4.1) is related to Fourier transformation (4.2) and (4.4) of the four-dimensional spacetime, eq.(4.1) is projected onto a plane wave function $e^{i \vec{p} \cdot \vec{r} / \beta-i E t / \alpha}$ of the four dimensional spacetime state vector and making integration, we get Schrödinger equation (4.6), Schrödinger equation (4.6) is reflecting both the particle property and the wave property, eq.(4.1) is only reflecting the nature of the particle, and that eq.(4.6) is turned into quantum mechanics just reflects the material particle property and wave property in the wave-particle duality for Schrödinger equation.

The complex square root function $\phi(\vec{p}, E)$ of the probability density of the classical particle in the complex field is transformed into $\psi(\vec{r}, t)$ through (4.3) and (4.5), which not only has the characteristics of the probability density state vector of the classical particle, but also has the characteristics of the wave, that is, which makes $\psi(\vec{r}, t)$ have the characteristics of the wave-particle duality state vector at the same time.

Thus, the basic interpretation problem of quantum mechanics has been debated for more than a century, e.g., the crisis of the dispute over the origin of the wave-particle duality of microscopic particles has been solved.

Consequently, eq.(4.1) and eq.(4.4), respectively, show the classical locality and quantum non-locality, $\phi(\vec{p}, E)$ (from the complex square root function of the classical probability density of a particle in the complex domain in eq.(4.3) ) and eq.(4.5) directly shows the classical locality and quantum non-locality.

Since in the current quantum mechanics, the third axiom presumption is the evolution axiom presumption: the evolution law of the wave function representing the state of the quantum isolated 
system before the measurement satisfies the wave equation (namely Schrödinger equation) relevant to the energy-momentum relation of classical mechanics. In the above study, we do not need the third axiom presumption to derive not only the entire contents of the third axiom presumption, but also more new physical contents.

It especially needs to point out: we extensively take the complex square root function of the probabilistic density function in the most general mathematical form, then the probabilistic density square root state vector function is obtained, which similarly take square root of Klein-Gorden equation to get very different Dirac equation, we have obtained a general quantum mechanical function of complex numbers, which is completely different from the classical probabilistic density function of real Numbers, and vice versa.

What is also special is that we have the classical statistical eigen-equation which is only different from Schrödinger equation of quantum mechanics in terms of the Fourier transformation of the plane wave, which is what makes the classical statistical mechanics eigen equation of particles become Schrödinger equation with wave-particle duality. So, they are related and consistent, and thus we have determined the strict correspondence relationships from the classical statistical mechanics to the quantum mechanics, or from the quantum mechanics to the classical statistical mechanics.

\section{Applications to different commutation relations and the first quantization}

Using the deduced operators $\hat{\vec{p}}=-i \hbar \nabla$ and $\hat{\mathrm{H}}(\hat{\vec{p}}, \vec{r})=i \hbar \partial / \partial t$, we have

$$
\begin{aligned}
& {[\hat{\mathrm{H}}(\hat{\vec{p}}, \vec{r}), t]=[i \hbar \partial / \partial t, t]=i \hbar,} \\
& {\left[x_{i}, \hat{p}_{j}\right]=\left[x_{i},-i \hbar \partial / \partial x_{j}\right]=i \hbar \delta_{i j},}
\end{aligned}
$$

and

$$
\left[x_{i}, x_{j}\right]=\left[\hat{p}_{i}, \hat{p}_{j}\right]=\left[x_{i}, t\right]=\left[\hat{p}_{i}, t\right]=0 .
$$

Therefore, for more operators, we naturally have

$$
\left[\hat{J}_{i}, \hat{x}_{j}\right]=i \hbar \varepsilon_{i j n} \hat{x}_{n},\left[\hat{p}_{k}, \hat{L}_{l}\right]=i \hbar \varepsilon_{k l m} \hat{p}_{m} .
$$

where angular momentum components $\hat{J}_{i}=\varepsilon_{i j k} \hat{x}_{j} \hat{p}_{k}, \varepsilon_{123}=-\varepsilon_{132}=1$.

In fact, when people deduce wave functions, operator expressions of mechanics quantities, operators' eigen-values and commutation relations of different operators, the first quantization of quantum theory has been achieved [15].

On the other hand, inserting the deduced momentum vector operator $\hat{\vec{p}}=-i \hbar \nabla$ into the derived Hamiltonian operator $\hat{\mathrm{H}}(\hat{\vec{p}}, \vec{r})=i \hbar \partial / \partial t$, we achieve $\hat{\mathrm{H}}(-i \hbar \nabla, \vec{r})=i \hbar \partial / \partial t$, using the deduced Hamiltonian operator $\hat{\mathrm{H}}(-i \hbar \nabla, \vec{r})=i \hbar \partial / \partial t$ to act a general wave function $\psi(\vec{r}, t)$, we derive a general Schrödinger equation

$$
i \hbar \frac{\partial}{\partial t} \psi(\vec{r}, t)=\hat{\mathrm{H}}(-i \hbar \nabla, \vec{r}) \psi(\vec{r}, t),
$$

which display that our studies are consistent with all the relevant investigations in this paper.

According to all the investigations above in this paper, we have done all the above these, consequently, we naturally show general quantum theory and its solutions to the two crisises of both wave-particle duality origin and the first quantization interpretations.

Quantum effects are actually caused by the wave-particle duality. Because if there is no matter wave property, i.e., there is not a plane wave with the integral transformation, it is not 
possible, at the same time, the presence of multiple points in the space, and multiple points all have probability waves, because probability wave can have distribution, matter probability wave function is used to describe wave-particle duality in current quantum theory, which is the basic hypothesis of quantum mechanics, but in our study is a natural deducing consequence.

\section{Summary and conclusions}

In classical statistical physics, the state of a microscopic particle or microscopic particle system is described by a real density function, we extensively find that the state of a microscopic particle or microscopic particle system can be described by a general complex function ( or call a state vector ) $\psi(\vec{r}, t)$ from Fourier integral transformation, with plane wave, of complex square root $\phi(\vec{p}, E)$ of the real density function of classical statistical mechanics. In fact, when we take the state $\psi(\vec{r}, t)$, we begin to enter a general description system that is different from the classical statistical mechanics system, namely the quantum mechanics system, which is, in some meaning, analogous to taking the square root of Klain-Gooden equation, we get a new system, Dirac equation Fermi system, which just means that we may say that quantum mechanics is a generalization mechanic theory of the complex square root of real density function of classical statistical mechanics, namely, we, for the first time, factually discover that quantum mechanics is a generalization mechanics theory of the complex square root of real density function of classical statistical mechanics, for short, quantum mechanics is just a generalization theory of the complex square root of classical statistical mechanics, which can be seen from the overall deductions in this paper, and it is both new physics and revolutionary discovery, further will affect people's deep philosophical thinking for modern physics development.

We naturally give inverse calculation of general matrix diagonalization for any observable physical quantity operator, and prove the general observable quantity operator must be Hermitian operator. In linear algebra, people have proved that any Hermitian matrix is diagonalizable, and the diagonalizable conditions are the sufficient and necessary conditions that the Hermitian matrixes acting on their eigen-vectors have eigenvalues.

Therefore, the above research is consistent with the known quantum mechanics theory, which is an important reason why we can generalize to quantum mechanics from classical statistical mechanics.

Not only do we deduce that the eigenvalues of all the operators are the values corresponding to the extreme values of their variational system, and find Hellmann-Feynman theorem is true only if the eigenvalues of all operators are the values corresponding to the extreme values of their variational system, and the rest values of the system are also the result of taking the extreme values for the variational system.

From the research in the second and third sections, we have now reduced the third axiom presumption ( evolution axiom presumption ) to evolution theorem, that is, the evolution theorem has been proved from the classical statistical mechanics.

When making 4-dimensional spacetime Fourier integral transformations (4.2) and (4.4) of eq.(4.1), that is, when eq.(4.1) is projected onto the state vector of the plane wave function $e^{i p \cdot r \cdot \beta-i E t / \alpha}$ of the four-dimensional spacetime and is integrated, we get Schrödinger equation (4.6), 
eq. (4.6) reflects both the particle nature of matter and the wave nature of matter. According to the exact deducing logic, in other words, eq.(4.1) only reflects the properties of particles, while eq.(4.6) is transformed into Schrödinger equation reflecting the wave-particle duality in quantum mechanics. The complex square root function $\phi(\vec{p}, E)$ of the probability density of the classical particle in the complex field is converted to $\psi(\vec{r}, t)$ by eq.(4.3) and eq.(4.5), it not only has the property of the probability density state vector of classical particles, but also has the property of the wave, which make $\psi(\vec{r}, t)$ have characteristics of wave-particle duality state vector at the same time.

However, the crisis of the fundamental interpretation of quantum mechanics, which has been debated for more than a century, isn't still solved, but, e.g., the crisis of the debates of the origin of the wave-particle duality of microscopic particles has been rigorously settled in this paper.

Therefore, eq.(4.1) and eq.(4.4), respectively, show the classical locality and quantum non-locality, $\phi(\vec{p}, E)$ in eq.(4.3) and $\psi(\vec{r}, t)$ in eq.(4.5) directly shows the classical locality and quantum non-locality respectively.

Using eq.(4.2), we deduce $\hat{\mathrm{H}}(\vec{p}, \vec{r})=i \hbar \partial / \partial t$, therefore, we discover that it is the existence of a projection weight factor $e^{-i t E / \hbar}$ that makes the Hamiltonian be written in operator form $\hat{\mathrm{H}}(\vec{p}, \vec{r})=i \hbar \partial / \partial t$, which just shows, in Schrödinger equation, taking derivative of the wave function is a partial derivative about time $t$ rather than a full derivative, i.e., it is the reason why people don't take the time derivative of space coordinates of the wave function. People haven't found out the reason for more than 80 years, because Schrödinger equation is still an axiom presumption up to now, but this paper deduces general Schrödinger equation via two kinds of general methods, anyway, up to now, it has not been rigorously derived in the most general way.

Because we have deduced wave functions, operator expressions of mechanics quantities, operators' eigenvalues and commutation relations of different operators, the first quantization of quantum theory not only has been achieved, but also we very naturally give the origin of the first quantization of quantum theory. Consequently, the crisis of the origin of the first quantization of quantum theory is naturally solved.

Therefore, we finally naturally deduce the very important three ones of the five axiom presumptions of quantum mechanics, then these three axiom presumptions are changed into three basic theorems of quantum mechanics. Consequently, we show general quantum theory and its solutions to crisises of origins of both wave-particle duality and the first quantization. Due to length limit of this paper, the studies on the other two axiom presumptions of quantum mechanics, origins and classifications of entanglements, wave collapse and so on would be given in following paper [35].

Acknowledgments: The work is supported by the U.S. Department of Energy, contract no. DE-AC02-05CH11231, NSF through grants PHY-08059, DOE through grant DEFG0291ER40681 and National Natural Science Foundation of China (No. 11875081). 


\section{References}

[1] R. A. Bagnold, Stochastic Methods in Quantum Mechanics, Dover Publications, 2005.

[2] David Griffiths, Introduction To Quantum Mechanics, Oversea Publishing House, 2004.

[3]Mc Call, Classical Mechanics: From Newton to Einstein: A Modern Introduction, Wiley, 2010.

[4] Georgy A. Martynov, Classical Statistical Mechanics, Springer Netherlands, 2011.

[5] B. L. van der Waerden, Sources of Quantum Mechanics, Dover Publications, 2007.

[6] Mark Beck, Quantum Mechanics: Theory and Experiment, Oxford University Press, 2012.

[7] Omnes, Roland, The Interpretation of Quantum Mechanics, Princeton University Press, 1988.

[8] Peter Mittelstaedt, The Interpretation of Quantum Mechanics and the Measurement Process, Cambridge University Press, 2004.

[9] Bohr N., Discussions with Einstein on Epistemological Problems in Atomic Physics. The Value of Knowledge: A Miniature Library of Philosophy. Marxists Internet Archive. Retrieved 2010-08-30. From Albert Einstein: Philosopher-Scientist (1949), publ. Cambridge University Press, 1949. Niels Bohr's report of conversations with Einstein.

[10]González A. M. Albert Einstein, Donostia International Physics Center. Retrieved 2010-08-30.

[11] Einstein (1969), A reprint of this book was published by Edition Erbrich in 1982.

[12] Momentum Transfer to a Free Floating Double Slit: Realization of Thought Experiment from the Einstein-Bohr Debates, L. Ph. H. Schmidt et al. Physical Review Letters Week ending 2013.

[13] C. Huang and Yong-Chang Huang, unification theory of classical statistical uncertainty relation and quantum uncertainty relation and its applications, Physics Letters A 375 (2011) 271.

[14] Bishop, Robert C. (2011). "Chaos, Indeterminism, and Free Will". In Kane, Robert. The Oxford Handbook of Free Will (Second ed.). Oxford, New York: Oxford University Press. p. 90. ISBN 978-0-19-539969-1. Retrieved 2013-02-04; Shmuel Friedland, Vlad Gheorghiu, and Gilad Gour, Universal Uncertainty Relations, Phys. Rev. Lett. 111, 230401 (2013) .

[15]J. J. Sakurai, J. Napolitano, Modern quantum mechanics, 2nd Edition, Addison-Wesley, 2011.

[16] Baldock, Robert John Nicholas, Classical Statistical Mechanics with Nested Sampling, Springer, 2017.

[17]Petrina, D. Y., Mathematical Foundations of Classical Statistical Mechanics, CRC Press, 1988.

[18] David McIntyre, Quantum Mechanics: A Paradigms Approach, Addison-Wesley, 2012.

[19] Abraham Pais, "Subtle is the Lord...", The Science and the Life of Albert Einstein, Oxford University Press, p.447-8, 1982.

[20] Niels Bohr in Albert Einstein: Philosopher-Scientist (P.Schilpp, Editor), p.199. Tudor, New York, 1949; N. BOHR, The Quantum Postulate and the Recent Development of Atomic Theory, Nature 121, 580-590, 1928.

[21] Mary Jo Nye, Quantum Theory's Silent Pioneer, Science, 326, No. 5957, 2009; Baggott, Jim E. "Complementarity and Entanglement". Beyond Measure: Modern Physics, Philosophy, and the Meaning of Quantum Theory. Oxford, New York: Oxford University Press. p. 203(2004). ISBN 0-19-852536-2. Retrieved 2013-02-04.

[22] Ilya Prigogine, History of Quantum Theory, Science, 221, 1983; Floyd Williams, Topics in Quantum Mechanics (Progress in Mathematical Physics) (V 27), Springer, Birkhouser, 2012.

[23] Davide Castelvecchi, Reimagining of Schrödinger's cat breaks quantum mechanics — and stumps physicists, Nature, 561, 446-447, 2018.

[24] Dustin Lazarovici \& Mario Hubert, How Quantum Mechanics can consistently describe the use of itself, Scientific Reports, 9, 1-8, 2019. 
[25] Flaminia Giacomini, Esteban Castro-Ruiz \& Časlav Brukner, Quantum mechanics and the covariance of physical laws in quantum reference frames, Nature Communications, 10(2019) 1.

[26] Gilad Gour, David Jennings, Iman Marvian, Quantum majorization and a complete set of entropic conditions for quantum thermodynamics, Nature Communications ,9, 1-9, 2018.

[27] Eric Scerri, Can quantum ideas explain chemistry's greatest icon? Nature, 565 (2019) 557.

[28] Magdalena Zych \& Časlav Brukner, Quantum formulation of the Einstein equivalence principle, Nature Physics, 14, 1027-1031, 2018.

[29]E. Zohar,Particle physics, Quantum simulation of fundamental physics,Nature, 534(2016) 480.

[30] Stephen L. Adler, et al., Is Quantum Theory Exact? Science, 325, No. 5938, 2009.

[31] A. Cho, Hawking's bid to save quantum theory from black holes, Science, 359(2018) 6382.

[32] A. L. Kuzemsky, Works of D. I. Blokhintsev and Development of Quantum Physics, Physics of Elementary Particles and Atomic Nuclei,39 (2008) 5--81.

[33] A. L. Kuzemsky, Probability, Information and Statistical Physics, Int. J. Theor. Phys., 55 (2016) 1378-1404.

[34] A. L. Kuzemsky, Fundamental Principles of Theoretical Physics and Concepts of Quasiaverages, Quantum Protectorate and Emergence, arXiv: 1207.6433v1; A. L. Kuzemsky, Temporal Evolution, Directionality of Time and Irreversibility, La Rivista del Nuovo Cimento, 41 (2018) 513-574.

[35] C. Huang, Yong-Chang Huang and Yi-You Nie, General Quantum Theory No Axiom Presumption: II ---- Measuring \& Identical Theorems, Origins \& Classifications of Entanglements and Solution to Crisis of Wave Collapse. Preprints 2020, 2020020092 (https:// www.preprints.org /manuscript/202002.0092/v1). 